\title{
ON THE LAGRANGIAN STRUCTURE OF THE DISCRETE ISOSPECTRAL AND ISOMONODROMIC TRANSFORMATIONS
}

\author{
ANTON DZHAMAY
}

\begin{abstract}
We study the Lagrangian properties of the discrete isospectral and isomonodromic dynamical systems. We generalize the Moser-Veselov approach to integrability of discrete isospectral systems via the refactorization of matrix polynomials to matrix rational functions with a simple divisor and consider in detail the case of two poles or, equivalently, of two elementary factors. In this case we establish, by explicitly writing down the Lagrangian, that the isospectral dynamic is Lagrangian. Next, we show how to make this Lagrangian time-dependent to obtain the equations of the isomonodromic dynamic. In some special cases such equations are known to reduce to the difference Painlevé equations. We show how to obtain the difference Painlevé V equation in that way, establishing that dPV can be written in the Lagrangian form.
\end{abstract}

\section{INTRODUCTION}

The theory of completely integrable systems and soliton equations is justly known for its rich and often unexpected connections with a wide range of other branches of mathematics and mathematical physics. In recent years its discrete variant, the theory of discrete completely integrable systems, started to attract a considerable amount of attention. This subject is a part of a more general field of discrete Lagrangian mechanics, which itself is gaining importance partly due to the development of new numerical algorithms based on discrete variational integrals, see, for example, the recent survey by J. Marsden and M. West, [MW01]. In a series of papers [Ves88, MV91, Ves91] A. Veselov and J. Moser showed that the discrete analogues of many classical integrable systems, e.g., the Neumann system and the spinning top, are related to the re-factorization transformation of certain matrix polynomials. Such a representation explains the integrability mechanism for these systems, since it is a discrete version of the Lax-pair representation. Hence, similarly to the continuous case, it can be used to integrate the system using theta functions. A large number of such examples can also be found in a recent encyclopedic book by Suris, [Sur03]. The relationship between matrix factorizations and integrable systems was observed earlier by Symes [Sym82], see also a related work by Deift et al, [DLT89].

The dynamic generated just by the re-factorization transformations is also known as the isospectral dynamic. Combining re-factorization with a shift in the spectral variable results in a different dynamics called isomonodromic, since it originates in the theory of isomonodromic transformations of systems of linear difference equations recently developed by A. Borodin [Bor04], see also [Kri04]. The theory of discrete isomonodromic transformations is important in part because, similarly to the continuous case, under certain conditions such transformations give rise to the discrete Painlevé equations from Sakai's hierarchy, [Sak01], thus clarifying the geometry of these equations.

In [Kri] I. Krichever conjectured that both the isospectral and the isomonodromic dynamic can (and maybe should) be considered from the Lagrangian point of view. In this paper we make a first step towards verifying this conjecture. We generalize the Moser-Veselov approach from the matrix polynomials to a large class of rational matrix functions on the Riemann sphere whose determinant divisor is simple. This generalization is important if one wants to consider discrete integrable systems that have higher-genus spectral curves. In addition, rational matrices of this type play an important role in Krichever's approach, [Kri04], to the isomonodromic deformations. Such matrices have natural factorization, where factors correspond to poles of the determinant divisor. In this paper we focus our attention on the simplest non-trivial case of two poles (and hence, two factors) and study in detail what happens when two factors are interchanged. At this point

2000 Mathematics Subject Classification. 39A10, 14H70, 70H06, 34M55.

Key words and phrases. discrete integrable systems; discrete Euler-Lagrange equations; difference Painlevé equations.

Supported in part by the University of Northern Colorado Summer 2006 SPARC Small Grant Assistance Program. 
there is no restrictions on the rank of the matrices. Our first result is that in this case both the isospectral and the isomonodromic dynamic is Lagrangian. Namely, we introduce a special coordinate system and then explicitly write down the expressions for the Lagrangian functions. Next, we restrict the rank to be 2 and verify that in this case the isomonodromic dynamic gives rise to the difference Painlevé equation dPV of the Sakai's hierarchy, thus establishing that dPV can be written in the Lagrangian form. This is our second result.

In the remainder of the introduction we give a detailed description of the setup of the problem and of our results.

1.1. Discrete Lagrangian systems. Continuous dynamical systems can be considered in the Lagrangian or in the Hamiltonian framework. Of those two, the Lagrangian approach is the one that naturally generalizes to the discrete case, see [Ves88, MV91, MW01]. Let $\mathcal{Q}$ be the configuration space of our system and let $n \in \mathbb{Z}$ be the discrete time parameter. In the continuous case the Lagrangian $\mathcal{L} \in \mathcal{F}(T \mathcal{Q})$ is a function on the tangent bundle of $\mathcal{Q}$. For the discrete case we need to change the point $(\mathbf{Q}, \dot{\mathbf{Q}})$ in the tangent space to the pair of points $(\mathbf{Q}, \tilde{\mathbf{Q}})$ in the configuration space itself. The Lagrangian then becomes a function on the square of the configuration space, $\mathcal{L} \in \mathcal{F}(\mathcal{Q} \times \mathcal{Q})$. The action functional $\mathcal{S}$ is then defined on the space of sequences $\left\{\mathbf{Q}_{k}\right\}, k \in \mathbb{Z}$ by the formal sum

$$
\mathcal{S}\left(\left\{\mathbf{Q}_{k}\right\}\right)=\sum_{k} \mathcal{L}\left(\mathbf{Q}_{k}, \mathbf{Q}_{k+1}\right),
$$

and the variational principle $\delta \mathcal{S}=0$ that selects the trajectories of the system, when written in a coordinate chart, takes the form of the discrete Euler-Lagrange equations

$$
\frac{\partial \mathcal{L}}{\partial \mathbf{Y}}(\underline{\mathbf{Q}}, \mathbf{Q})+\frac{\partial \mathcal{L}}{\partial \mathbf{X}}(\mathbf{Q}, \widetilde{\mathbf{Q}})=0
$$

where we use the notation $\underset{\mathbf{Q}}{\mathbf{Q}}=\mathbf{Q}_{k-1}, \mathbf{Q}=\mathbf{Q}_{k}$, and $\widetilde{\mathbf{Q}}=\mathbf{Q}_{k+1}$. These equations then implicitly define the map (or, more precisely, a correspondence) $\widetilde{\mathbf{Q}}=\phi(\underset{\mathbf{Q}}{\mathbf{Q}})$, which in turn defines the shift (or step) map $\Phi: \mathcal{Q} \times \mathcal{Q} \rightarrow \mathcal{Q} \times \mathcal{Q}$ by $\Phi(\underset{\mathbf{Q}}{\mathbf{Q}} \mathbf{Q})=(\mathbf{Q}, \widetilde{\mathbf{Q}})$. The map $\Phi$ is symplectic w.r.t. the 2 -form $\sigma=\frac{\partial^{2} \mathcal{L}}{\partial \mathbf{X} \partial \mathbf{Y}} d \mathbf{X} \wedge d \mathbf{Y}$ on $\mathcal{Q} \times \mathcal{Q}$. Alternatively, using the discrete version of the Legendre transform by defining the conjugated momentum $\mathbf{P}=\frac{\partial \mathcal{L}}{\partial \mathbf{Y}}(\mathbf{Q}, \mathbf{Q}) \in T_{\mathbf{Q}}^{*} \mathcal{Q}$, we see that the discrete Euler-Lagrange equations are equivalent to the system

$$
\left\{\begin{array}{l}
\mathbf{P}=-\frac{\partial \mathcal{L}}{\partial \mathbf{X}}(\mathbf{Q}, \widetilde{\mathbf{Q}}) \\
\widetilde{\mathbf{P}}=\frac{\partial \mathcal{L}}{\partial \mathbf{Y}}(\mathbf{Q}, \widetilde{\mathbf{Q}}),
\end{array}\right.
$$

where the first equation follows from (1.1) and is an implicit equation for $\widetilde{\mathbf{Q}}=\widetilde{\mathbf{Q}}(\mathbf{Q}, \mathbf{P})$. Hence we get a $\operatorname{map} \Psi: T_{\mathcal{Q}}^{*} \rightarrow T_{\widetilde{\mathcal{Q}}}^{*}, \Psi(\mathbf{Q}, \mathbf{P})=(\widetilde{\mathbf{Q}}, \widetilde{\mathbf{P}})$ which is symplectic w.r.t. the standard symplectic structure. In what follows by the equations of motion of a discrete Lagrangian system we mean either (1.1) or (1.2), with the corresponding discrete dynamics given by the maps $\Phi$ or $\Psi$ respectively.

1.2. Discrete integrable systems. The Moser-Veselov approach to the discrete integrable systems is based on the discrete version of the Lax pair representation and can be briefly described as follows. Given a discrete dynamical system, we look for a class $\mathcal{P}$ of matrix polynomials $\mathbf{L}(z)$ in a spectral variable $z$ and a parameterization map $\eta: \mathcal{Q} \times \mathcal{Q} \rightarrow \mathcal{P}$, defined on some dense open set, such that:

(i) there is a well-defined factorization rule $\mathbf{L}(z)=\mathbf{L}_{1}(z) \mathbf{L}_{2}(z)$, where the ordering is important, such that the re-factorized matrix $\tilde{L}$ obtained by the interchanging the order of the factors, $\tilde{\mathbf{L}}(z)=\mathbf{L}_{2}(z) \mathbf{L}_{1}(z)$, is again in $\mathcal{P}$ and hence can be written as $\tilde{\mathbf{L}}(z)=\tilde{\mathbf{L}}_{1}(z) \tilde{\mathbf{L}}_{2}(z)$, this rule defines the re-factorization map $R: \mathcal{P} \rightarrow \mathcal{P}$

(ii) under the parameterization $\eta$ the re-factorization map $R$ corresponds to the shift map $\Phi$ of our discrete dynamical system. 
Note that the re-factorization map can also be written in the form

$$
\tilde{\mathbf{L}}=\mathbf{M}^{-1} \mathbf{L M},
$$

where $\mathbf{M}=\mathbf{L}_{1}(z)$. Equation (1.3) is known as a discrete Lax pair representation of the system. Similarly to the continuous case, finding such a representation shows that the dynamic of the system is isospectral. Therefore, it preserves the spectral curve $\Gamma$ of the operator $\mathbf{L}(z)$, which implies the integrability of the system and also makes it possible to obtain the $\theta$-function formulas for solutions of the system in the usual way, see [Ves91].

1.3. Rational matrices anzats. Let us now allow $\mathbf{L}(z)$ to be a meromorphic $r \times r$-matrix function on the Riemann sphere with the poles $z_{1}, \ldots, z_{n}$. We restrict our attention to the matrices that are generic in the following sense:

(i) all poles $z_{i}$ of $\mathbf{L}(z)$ are simple;

(ii) the divisor of $\mathbf{L}(z)$ is simple as well, where by the divisor of $\mathbf{L}(z)$ we mean the divisor of its determinant function, $\mathcal{D}=(\mathbf{L}(z))=(\operatorname{det} \mathbf{L}(z))=\sum_{i} z_{i}-\sum_{j} \zeta_{j}$. This is equivalent to the condition that the residue matrices $\mathbf{L}_{k}:=\operatorname{res}_{z_{k}} \mathbf{L}(z)$ are of rank one.

For a fixed divisor $\mathcal{D}$ we denote the space of all such matrices by $\mathcal{M}_{r}^{D}$.

Without any loss of generality we can further restrict out attention to the case $z_{0}=\infty \notin \mathcal{D}$ and $\mathbf{L}_{0}=$ $\lim _{z \rightarrow \infty} \mathbf{L}(z)$ is invertible and diagonalizable. Any such matrix $\mathbf{L}(z)$ has two different representations, additive:

$$
\mathbf{L}(z)=\mathbf{L}_{0}+\sum_{k} \frac{\mathbf{L}_{k}}{z-z_{k}}
$$

and multiplicative:

$$
\mathbf{L}(z)=\prod_{k}\left(\mathbf{A}+\frac{\mathbf{G}_{k}}{z-z_{k}}\right)
$$

where $\mathbf{G}_{k}$ is a matrix of rank one and $\mathbf{A}^{k}=\mathbf{L}_{0}$; we are mainly interested in the multiplicative representation. We call the factors $\mathbf{B}_{i}^{\mathbf{A}}(z)=\left(\mathbf{A}+\frac{\mathbf{G}_{i}}{z-z_{i}}\right)$ in the multiplicative representation the elementary divisors of $\mathbf{L}(z)$. Note that the ordering of the poles determines the ordering of the factors in the multiplicative representation, which is what we need to define the re-factorization map.

In this paper we restrict our attention to the two-pole case. However, since any permutation is a composition of elementary transposition, any re-factorization transformation is generated by a sequence of transformations that we consider below. Thus, we expect our results to hold in the general case as well, but this question will be considered elsewhere.

1.4. The re-factorization transformation and the isospectral discrete dynamical system. Let us now fix the divisor $\mathcal{D}=z_{1}+z_{2}-\zeta_{1}-\zeta_{2}$, where all four points are finite and distinct, and consider the re-factorization map $R: \mathcal{M}_{r}^{D} \rightarrow \mathcal{M}_{r}^{D}$ given by

$$
\mathbf{L}(z)=\mathbf{B}_{1}^{\mathbf{A}}(z) \mathbf{B}_{2}^{\mathbf{A}}(z) \mapsto \tilde{\mathbf{L}}(z)=\mathbf{B}_{2}^{\mathbf{A}}(z) \mathbf{B}_{1}^{\mathbf{A}}(z)=\tilde{\mathbf{B}}_{1}^{\mathbf{A}}(z) \tilde{\mathbf{B}}_{2}^{\mathbf{A}}(z) .
$$

We want to determine whether there is a natural discrete dynamical system for which this map is a discrete Lax pair representation, and if so, what is the Lagrangian of this system. Note that this setting is rather general, since no restrictions on the rank $r$ are imposed. First it is necessary to identify a configuration space $\mathcal{Q}$ such that there is a parameterization map $\eta: \mathcal{Q} \times \mathcal{Q} \rightarrow \mathcal{M}_{r}^{\mathcal{D}}$ satisfying the following diagram:

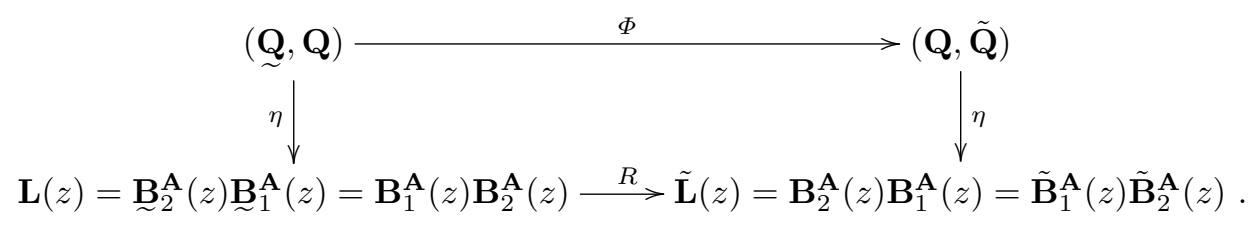

From this diagram it is clear that half the data in $\mathbf{L}(z)$ should come from $\mathbf{Q}$ and half should come from $\mathbf{Q}$. Moreover, this data should be of the same type to be compatible with the shift map $\Phi$. We know that the 
elementary divisors $\mathbf{B}_{i}^{\mathbf{A}}(z)$ completely determine $\mathbf{L}(z)$, and each elementary divisor is in turn determined by the rank-one matrix $\mathbf{G}_{i}=\mathbf{p}_{i} \mathbf{q}_{i}^{\dagger}$, where $\mathbf{p}_{i}$ and $\mathbf{q}_{i}^{\dagger}$ are defined up to a common scaling constant, and this constant can be recovered from the divisor $\mathcal{D}$. Thus, $\mathbf{L}(z)$ is completely determined by either $\underset{\sim}{\mathbf{p}} 1, \sim_{2}^{\mathbf{p}_{2}},{\underset{\mathbf{q}}{1}}_{1}^{\dagger},{\underset{\sim}{\mathbf{q}}}_{2}^{\dagger}$ or $\mathbf{p}_{1}, \mathbf{p}_{2}, \mathbf{q}_{1}^{\dagger}, \mathbf{q}_{2}^{\dagger}$. In view of that we take $\underset{\sim}{\mathbf{Q}}=({\underset{\sim}{\mathbf{p}}}_{1}, \underbrace{\mathbf{q}}_{2}), \mathbf{Q}=\left(\mathbf{p}_{1}, \mathbf{q}_{2}^{\dagger}\right)$. A priori $\mathbf{Q} \in \mathbb{C}^{r} \times\left(\mathbb{C}^{r}\right)^{\dagger}$, but in fact the resulting expressions are homogeneous in $\mathbf{p}_{i}, \mathbf{q}_{i}^{\dagger}$, and so the correct configuration space is $\mathcal{Q}=\mathbb{P}^{r-1} \times\left(\mathbb{P}^{r-1}\right)^{\dagger}$. In Theorem 3.1 we give an explicit description of the parameterization map $\eta: \mathcal{Q} \times \mathcal{Q} \rightarrow \mathcal{M}_{r}^{\mathcal{D}}$, compute the corresponding equations of motion, and show that these equations of motion are the discrete Euler-Lagrange equations with the Lagrangian function $\mathcal{L}$ given by

$$
\begin{aligned}
\mathcal{L}(\mathbf{X}, \mathbf{Y})= & \left(z_{2}-z_{1}\right) \log \left(\mathbf{x}_{2}^{\dagger} \mathbf{x}_{1}\right)+\left(z_{1}-\zeta_{2}\right) \log \left(\mathbf{x}_{2}^{\dagger} \mathbf{A}^{-1} \mathbf{y}_{1}\right)+ \\
& \left(\zeta_{2}-\zeta_{1}\right) \log \left(\mathbf{y}_{2}^{\dagger} \mathbf{A}^{-2} \mathbf{y}_{1}\right)+\left(\zeta_{1}-z_{2}\right) \log \left(\mathbf{y}_{2}^{\dagger} \mathbf{A}^{-1} \mathbf{x}_{1}\right) .
\end{aligned}
$$

1.5. Discrete Painlevé equations and the isomonodromic transformations of the systems of linear difference equations. Another natural discrete dynamics that can be considered on our space of matrices is the isomonodromic discrete dynamical system. One of the reasons this system is interesting is its relationship to the theory of the discrete Painlevé equations.

The recent surge of interest in the discrete version of the famous Painlevé equations is in part due to the fact that these equations appear in the calculation of discrete gap probabilities in the theory of (determinantal) Random Point Processes, [Bor03, BD02]. In addition, H. Sakai in [Sak01] described a very elegant and purely geometric approach to the discrete Painlevé equations using the Cremona action on the algebraic surfaces. More information about the current progress in the theory of discrete Painlevé equations can be found in [GR04].

In the continuous case there is a well-known relationship between the isomonodromic transformations of the flat meromorphic connections on the Riemann sphere and the Painlevé equations. Thus, it is natural to expect that the discrete Painlevé equations should be related to the isomonodromic deformations of matrix linear difference equations. However, there is a serious obstacle - the notion of monodromy for a differential equation has no obvious generalization to a difference equation, and only recently some significant progress was made in this direction. Recall that the general theory of matrix linear differential equations

$$
\boldsymbol{\Psi}(z+1)=\mathbf{L}(z) \boldsymbol{\Psi}(z)
$$

goes back the works of George Birkhoff, [Bir11]. First step in Birkhoff's approach was to use a special gauge transformation to clear all poles of $\mathbf{L}(z)$ and make it a polynomial in $z$. Note that as a result we get a pole of higher order at infinity. Next, Birkhoff showed that there are two canonical meromorphic solutions $\boldsymbol{\Psi}_{l}(z)$ and $\boldsymbol{\Psi}_{r}(z)$ that have the prescribed asymptotic behavior for $\Re(z) \ll 0$ and $\Re(z) \gg 0$ respectively. Then the analogue of the monodromy map is just the connection matrix $\mathbf{C}$ of these solutions, $\mathbf{C}(z)=\mathbf{\Psi}_{r}^{-1}(z) \mathbf{\Psi}_{l}(z)$. Birkhoff also showed that in this situation the isomonodromic transformations $\mathbf{L}(z) \mapsto \tilde{\mathbf{L}}(z)$ that preserve $\mathbf{C}(z)$ are given by

$$
\tilde{\mathbf{L}}(z)=\mathbf{R}(z+1) \mathbf{L}(z) \mathbf{R}^{-1}(z),
$$

where $\mathbf{R}(z)$ is a rational matrix. A. Borodin, in [Bor04], constructed a general theory of such transformations for polynomial $\mathbf{L}(z)$ and showed that it give rise to the difference Schlesinger equations. These equations, when the the space of parameters is two-dimensional, can in turn be reduced to the difference Painlevé equations. In a follow-up paper [AB06], D. Arinkin and A. Borodin showed, using a more geometric language of $d$-connections, that for some special cases Sakai's surfaces can be identified with the moduli space of such $d$-connections and that the isomonodromic transformations can then be though of as the elementary modifications of $d$-connections, which are in turn given by the difference Painlevé equations (examples considered in this paper are $\mathrm{dPV}$ and $\mathrm{dPVI}$ ). This result helps to explain the geometry behind the difference isomonodromy-Painlevé correspondence. Recently, Arinkin and Borodin found the description for the $\tau$-function of the discrete isomonodromy transformations for both polynomial and rational cases, see [AB07].

A different approach to the notion of the monodromy of a linear difference equation was suggested by I. Krichever in [Kri04]. In this approach the matrix $\mathbf{L}(z)$ belongs to the same anzats as we consider in the present paper - it is regular at infinity, all of its poles $z_{i}$ are finite and simple, and $\operatorname{res}_{z_{i}} \mathbf{L}(z)$ are of rank one. For such matrices Krichever introduced the notion of a local monodromy that can be thought of as a monodromy corresponding to the path around a pole, and also constructed the isomonodromy 
transformations, that again have the form (1.6). He also showed how to generalize this theory from rational to elliptic functions.

In the present paper we consider a special case of the transformation (1.6) that has the form

$$
\mathbf{L}(z)=\mathbf{B}_{1}^{\mathbf{A}}(z) \mathbf{B}_{2}^{\mathbf{A}}(z) \mapsto \tilde{\mathbf{L}}(z)=\tilde{\mathbf{B}}_{1}^{\mathbf{A}}(z) \tilde{\mathbf{B}}_{2}^{\mathbf{A}}(z)=\mathbf{B}_{2}^{\mathbf{A}}(z+1) \mathbf{B}_{1}^{\mathbf{A}}(z)=\mathbf{B}_{2}^{\mathbf{A}}(z+1) \mathbf{L}(z)\left(\mathbf{B}_{2}^{\mathbf{A}}(z)\right)^{-1},
$$

where $\mathbf{B}_{i}^{\mathbf{A}}(z)$ are the elementary divisors defined earlier. We show that, similarly to the isospectral case, these transformations can be written in the Lagrangian form. The main new feature of the isomonodromic approach is the fact that such transformation changes the divisor $D$ to the divisor $\tilde{D}$, where $\tilde{z}_{1}=z_{1}, \tilde{\zeta}_{1}=\zeta_{1}$, $\tilde{z}_{2}=z_{2}-1$, and $\tilde{\zeta}_{2}=\zeta_{2}-1$. Thus we need to make the Lagrangian $\mathcal{L}$ time-dependent by putting $z_{2}(t)=z_{2}-t$ and $\zeta_{2}(t)=\zeta_{2}-t$ :

$$
\begin{aligned}
\mathcal{L}(\mathbf{X}, \mathbf{Y}, t)= & \left(z_{2}(t)-z_{1}\right) \log \left(\mathbf{x}_{2}^{\dagger} \mathbf{x}_{1}\right)+\left(z_{1}-\zeta_{2}(t)\right) \log \left(\mathbf{x}_{2}^{\dagger} \mathbf{A}^{-1} \mathbf{y}_{1}\right)+ \\
& \left(\zeta_{2}(t)-\zeta_{1}\right) \log \left(\mathbf{y}_{2}^{\dagger} \mathbf{A}^{-2} \mathbf{y}_{1}\right)+\left(\zeta_{1}-z_{2}(t)\right) \log \left(\mathbf{y}_{2}^{\dagger} \mathbf{A}^{-1} \mathbf{x}_{1}\right) .
\end{aligned}
$$

The time-dependent discrete Euler-Lagrange equations

$$
\frac{\partial \mathcal{L}}{\partial \mathbf{Y}}\left(\mathbf{Q}_{k-1}, \mathbf{Q}_{k}, k-1\right)+\frac{\partial \mathcal{L}}{\partial \mathbf{X}}\left(\mathbf{Q}_{k}, \mathbf{Q}_{k+1}, k\right)=0
$$

then describe the isomonodromic dynamics (1.7). Finally, we verify, essentially following [AB06], that in the rank-two case equation (1.7), when written in the so-called spectral coordinates, reduces to the difference Painlevé equation dPV of the Sakai's hierarchy, thus establishing that this equation can be written in the Lagrangian form.

1.6. Organization of the paper. In Section 2 we study properties the elementary divisors, and obtain the description of the re-factorization map. In Section 3 we establish the Lagrangian structure of the isospectral dynamics, and in Section 4 we extend this result to the isomonodromic case.

\section{Elementary Divisors}

In representing rational matrix functions in the multiplicative form we take each factor to be a matrix of the following simple type.

Definition 2.1. An elementary divisor with the simple pole at $z_{i}$ is a matrix of the form $\mathbf{B}_{i}^{\mathbf{A}}(z)=\mathbf{A}+\frac{\mathbf{G}_{i}}{z-z_{i}}$, where $\mathbf{G}_{i}$ is a matrix of rank one and $\mathbf{A}$ is some fixed constant non-degenerate matrix (which is usually taken to be diagonal).

In this section we describe certain useful properties of elementary divisors, and also explain our normalization conventions.

2.1. Rank-one matrices and normalization. Let us first make some remarks about matrices of rank one. Any such matrix has the form $\mathbf{G}=\mathbf{p q}^{\dagger}$ for some column vector $\mathbf{p}$ and some row vector $\mathbf{q}^{\dagger}$, where the vectors $\mathbf{p}$ and $\mathbf{q}^{\dagger}$ are defined up to a common scaling constant. To explicitly keep track of such scaling constants during computations we need to normalize these vectors in some way.

$\triangleleft$ Notation: Given the choice of a normalization, we denote by [v] the normalization of a vector $\mathbf{v}$ and by $\nu(\mathbf{v})$ its normalization constant w.r.t. this normalization. Thus, $\mathbf{v}=\nu(\mathbf{v})[\mathbf{v}]$. We also use the notation $[\mathbf{v}]$ for the normalized vectors. Hence, any matrix of rank one can be written as $\mathbf{G}=\mathbf{p q}^{\dagger}=\nu(\mathbf{G})[\mathbf{p}]\left[\mathbf{q}^{\dagger}\right]=\lambda[\mathbf{p}]\left[\mathbf{q}^{\dagger}\right]$, where $\lambda=\nu(\mathbf{G})=\nu(\mathbf{p}) \nu\left(\mathbf{q}^{\dagger}\right)$ is the normalization constant for $\mathbf{G}$. $\triangleright$

For our purposes it is most convenient to work with linear normalizations. Such normalizations have the property that any linear relation among the normalized vectors implies the same linear relation for the coefficients; if $a[\mathbf{u}]=\sum_{k} b_{k}\left[\mathbf{v}_{k}\right]$, then $a=\sum_{k} b_{k}$. For example, the normalizations

(a) $\sum_{i}(\mathbf{p})^{i}=\sum_{j}\left(\mathbf{q}^{\dagger}\right)_{j}=1$,

(b) $(\mathbf{p})^{i}=\left(\mathbf{q}^{\dagger}\right)_{j}=1$ for some choice of indexes $i$ and $j$ 
satisfy this requirement.

For an elementary divisor we can use one of the following three natural normalizations:

$$
\mathbf{B}_{i}^{\mathbf{A}}(z)=\mathbf{A}+\frac{\lambda_{i}\left[\mathbf{p}_{i}\right]\left[\mathbf{q}_{i}^{\dagger}\right]}{z-z_{i}}=\mathbf{A}\left(\mathbf{1}+\frac{\lambda_{i}^{\mathbf{p}}\left[\mathbf{A}^{-1} \mathbf{p}_{i}\right]\left[\mathbf{q}_{i}^{\dagger}\right]}{z-z_{i}}\right)=\left(\mathbf{1}+\frac{\lambda_{i}^{\mathbf{q}^{\dagger}}\left[\mathbf{p}_{i}\right]\left[\mathbf{q}_{i}^{\dagger} \mathbf{A}^{-1}\right]}{z-z_{i}}\right) \mathbf{A}
$$

where the superscript $\mathbf{p}$ in $\lambda_{i}^{\mathbf{p}}$ indicates that instead of normalizing $\mathbf{p}$ we normalize $\mathbf{A}^{-1} \mathbf{p}$; similarly, $\lambda_{i}^{\mathbf{q}^{\dagger}}$ corresponds to the normalization of $\mathbf{q}_{i}^{\dagger} \mathbf{A}^{-1}$.

2.2. Properties of elementary divisors. The following Lemma is a key technical tool for working with elementary divisors.

Lemma 2.1. Let $\mathbf{B}_{i}^{\mathbf{A}}(z)=\mathbf{A}+\frac{\mathbf{G}_{i}}{z-z_{i}}$ and define $\zeta_{i}$ by the equation $\operatorname{tr}\left(\mathbf{G}_{i} \mathbf{A}^{-1}\right)=z_{i}-\zeta_{i}$. Then the following holds.

(i) $\operatorname{det} \mathbf{B}_{i}^{\mathbf{A}}(z)=\frac{z-\zeta_{i}}{z-z_{i}} \operatorname{det} \mathbf{A}$ and $\left(\mathbf{B}_{i}^{\mathbf{A}}(z)\right)^{-1}=\mathbf{A}^{-\mathbf{1}}\left(\mathbf{A}-\frac{\mathbf{G}_{i}}{z-\zeta_{i}}\right) \mathbf{A}^{-1}$.

(ii) Knowing how $\mathbf{B}_{i}^{\mathbf{A}}(z)$ operates on row (resp. column) vectors and also knowing the column (resp. row) vector of the rank one part allows us to determine $\mathbf{B}_{i}^{\mathbf{A}}(z)$ :

$$
\begin{aligned}
& \text { - if } \mathbf{v}=\mathbf{B}_{i}^{\mathbf{A}}(z) \mathbf{w} \text {, then } \mathbf{G}_{i}=\mathbf{A}\left(\left(z_{i}-z\right) \frac{\mathbf{w}}{\mathbf{q}_{i}^{\dagger} \mathbf{w}}+\left(z-\zeta_{i}\right) \frac{\mathbf{A}^{-1} \mathbf{v}}{\mathbf{q}_{i}^{\dagger} \mathbf{A}^{-1} \mathbf{v}}\right) \mathbf{q}_{i}^{\dagger} \\
& \text { - if } \mathbf{v}^{\dagger}=\mathbf{w}^{\dagger} \mathbf{B}_{i}^{\mathbf{A}}(z) \text {, then } \mathbf{G}_{i}=\mathbf{p}_{i}\left(\left(z_{i}-z\right) \frac{\mathbf{w}_{i}^{\dagger}}{\mathbf{w}^{\dagger} \mathbf{p}_{i}}+\left(z-\zeta_{i}\right) \frac{\mathbf{v}^{\dagger} \mathbf{A}^{-1}}{\mathbf{v}^{\dagger} \mathbf{A}^{-1} \mathbf{p}_{i}}\right) \mathbf{A} \text {. }
\end{aligned}
$$

Proof. To prove part (i), note that

$$
\operatorname{det} \mathbf{B}_{i}^{\mathbf{A}}(z)=\operatorname{det}\left(\mathbf{1}+\frac{\mathbf{G}_{i} \mathbf{A}^{-1}}{\left(z-z_{i}\right)}\right) \operatorname{det} \mathbf{A}=\left(1+\frac{\operatorname{tr}\left(\mathbf{G}_{i} \mathbf{A}^{-1}\right)}{\left(z-z_{i}\right)}\right) \operatorname{det} \mathbf{A}=\frac{z-\zeta_{i}}{z-z_{i}} \operatorname{det} \mathbf{A},
$$

since $\mathbf{G}_{i} \mathbf{A}^{-1}$ is a matrix of rank one. The formula for the inverse matrix can be checked by the direct calculation.

To establish part (ii) we normalize the elementary divisor. Then, using the linearity property of the normalization, the equation $\mathbf{v}=\mathbf{B}_{i}^{\mathbf{A}}(z) \mathbf{w}$ can be written as

$$
\left[\mathbf{A}^{-1} \mathbf{v}\right]=\left[[\mathbf{w}]+\frac{\lambda_{i}^{\mathbf{p}}\left[\mathbf{A}^{-1} \mathbf{p}_{i}\right]\left[\mathbf{q}_{i}^{\dagger}\right][\mathbf{w}]}{z-z_{i}}\right]=\frac{[\mathbf{w}]\left(z-z_{i}\right)+\lambda_{i}^{\mathbf{p}}\left[\mathbf{A}^{-1} \mathbf{p}_{i}\right]\left[\mathbf{q}_{i}^{\dagger}\right][\mathbf{w}]}{\left(z-z_{i}\right)+\lambda_{i}^{\mathbf{p}}\left[\mathbf{q}_{i}^{\dagger}\right][\mathbf{w}]}
$$

Multiplying both sides by $\left[\mathbf{q}_{i}^{\dagger}\right]$ gives $\left(z-z_{i}\right)+\lambda_{i}^{\mathbf{p}}\left[\mathbf{q}_{i}^{\dagger}\right][\mathbf{w}]=\frac{\left[\mathbf{q}_{i}^{\dagger}\right][\mathbf{w}]\left(z-\zeta_{i}\right)}{\left[\mathbf{q}_{i}^{\dagger}\right]\left[\mathbf{A}^{-1} \mathbf{v}\right]}$, and so

$$
\mathbf{G}_{i}=\mathbf{A}\left(\lambda_{i}^{\mathbf{p}}\left[\mathbf{A}^{-1} \mathbf{p}_{i}\right]\left[\mathbf{q}_{i}^{\dagger}\right]\right)=\mathbf{A}\left(\frac{\left(z_{i}-z\right)[\mathbf{w}]\left[\mathbf{q}_{i}^{\dagger}\right]}{[\mathbf{w}]\left[\mathbf{q}_{i}^{\dagger}\right]}+\frac{\left(z-\zeta_{i}\right)\left[\mathbf{A}^{-1} \mathbf{v}\right]\left[\mathbf{q}_{i}^{\dagger}\right]}{\left[\mathbf{q}_{i}^{\dagger}\right]\left[\mathbf{A}^{-1} \mathbf{v}\right]}\right) .
$$

Since the expression in the parentheses is homogeneous, we can remove the normalization brackets to get the desired result. Second formula is obtained in a similar way.

2.3. The re-factorization transformation. Consider now the following question. Let

$$
\mathbf{B}_{2}^{\mathbf{A}}(z) \mathbf{B}_{1}^{\mathbf{A}}(z)=\widetilde{\mathbf{B}}_{1}^{\mathbf{A}}(z) \widetilde{\mathbf{B}}_{2}^{\mathbf{A}}(z)
$$

where $\mathbf{B}_{i}^{\mathbf{A}}(z)$ and $\widetilde{\mathbf{B}}_{i}^{\mathbf{A}}(z)$ are elementary divisors with the simple poles at $z_{i}$. What are the relationships between the vectors that form their rank-one parts? To begin with, note that taking the determinant results in the equation $\frac{\left(z-\zeta_{i}\right)\left(z-\zeta_{2}\right)}{\left(z-z_{i}\right)\left(z-z_{2}\right)}=\frac{\left(z-\tilde{\zeta_{1}}\right)\left(z-\tilde{\zeta}_{2}\right)}{\left(z-\tilde{z_{1}}\right)\left(\tilde{\zeta}_{\tilde{z}}\right)}$, where $\tilde{z}_{i}=z_{i}$ by definition, and so we must have either $\tilde{\zeta}_{i}=\zeta_{i}$ (the general case) or $\tilde{\zeta}_{1}=\zeta_{2}$ and $\tilde{\zeta}_{2}=\zeta_{1}$ (which is a special case, since it requires a non-trivial relationship between the poles and the rank-one parts of the elementary divisors,

$$
z_{2}-z_{1}=\operatorname{tr}\left(\tilde{\mathbf{G}}_{2} \mathbf{A}^{-1}\right)-\operatorname{tr}\left(\mathbf{G}_{1} \mathbf{A}^{-1}\right)=\operatorname{tr}\left(\mathbf{G}_{2} \mathbf{A}^{-1}\right)-\operatorname{tr}\left(\tilde{\mathbf{G}}_{1} \mathbf{A}^{-1}\right) .
$$


The Theorem below explains the general case, and the special case can be considered in exactly the same way.

Theorem 2.2. Let $\mathbf{B}_{2}^{\mathbf{A}}(z) \mathbf{B}_{1}^{\mathbf{A}}(z)=\widetilde{\mathbf{B}}_{1}^{\mathbf{A}}(z) \widetilde{\mathbf{B}}_{2}^{\mathbf{A}}(z)$ and $\tilde{\zeta}_{i}=\zeta_{i}$. Then the following holds.

(i) The vectors $\mathbf{p}_{i}, \tilde{\mathbf{p}}_{i}, \mathbf{q}_{i}^{\dagger}$, $\tilde{\mathbf{q}}_{i}^{\dagger}$ are related by

$$
\begin{array}{ll}
{\left[\tilde{\mathbf{p}}_{1}\right]=\left[\mathbf{B}_{2}^{\mathbf{A}}\left(z_{1}\right) \mathbf{p}_{1}\right]=\left[\mathbf{A} \widetilde{\mathbf{B}}_{2}^{\mathbf{A}}\left(\zeta_{1}\right) \mathbf{A}^{-1} \mathbf{p}_{1}\right]} & {\left[\mathbf{p}_{2}\right]=\left[\widetilde{\mathbf{B}}_{1}^{\mathbf{A}}\left(z_{2}\right) \tilde{\mathbf{p}}_{2}\right]=\left[\mathbf{A B}_{1}^{\mathbf{A}}\left(\zeta_{2}\right) \mathbf{A}^{-1} \tilde{\mathbf{p}}_{2}\right]} \\
{\left[\mathbf{q}_{1}^{\dagger}\right]=\left[\tilde{\mathbf{q}}_{1}^{\dagger} \widetilde{\mathbf{B}}_{2}^{\mathbf{A}}\left(z_{1}\right)\right]=\left[\tilde{\mathbf{q}}_{1}^{\dagger} \mathbf{A}^{-1} \mathbf{B}_{2}^{\mathbf{A}}\left(\zeta_{1}\right) \mathbf{A}\right]} & {\left[\tilde{\mathbf{q}}_{2}^{\dagger}\right]=\left[\mathbf{q}_{2}^{\dagger} \mathbf{B}_{1}^{\mathbf{A}}\left(z_{2}\right)\right]=\left[\mathbf{q}_{2}^{\dagger} \mathbf{A}^{-1} \widetilde{\mathbf{B}}_{1}^{\mathbf{A}}\left(\zeta_{2}\right) \mathbf{A}\right] .}
\end{array}
$$

(ii) The vectors $\mathbf{p}_{1}, \tilde{\mathbf{p}}_{1}, \mathbf{q}_{2}^{\dagger}, \tilde{\mathbf{q}}_{2}^{\dagger}$ completely determine the elementary divisors $\mathbf{B}_{i}^{\mathbf{A}}(z), \tilde{\mathbf{B}}_{i}^{\mathbf{A}}(z)$ via

$$
\begin{aligned}
& \mathbf{G}_{1}=\left(\left(z_{1}-z_{2}\right) \frac{\mathbf{p}_{1} \mathbf{q}_{2}^{\dagger}}{\mathbf{q}_{2}^{\dagger} \mathbf{p}_{1}}+\left(z_{2}-\zeta_{1}\right) \frac{\mathbf{p}_{1} \tilde{\mathbf{q}}_{2}^{\dagger} \mathbf{A}^{-1}}{\tilde{\mathbf{q}}_{2}^{\dagger} \mathbf{A}^{-1} \mathbf{p}_{1}}\right) \mathbf{A}, \\
& \tilde{\mathbf{G}}_{1}=\left(\left(z_{1}-\zeta_{2}\right) \frac{\tilde{\mathbf{p}}_{1} \mathbf{q}_{2}^{\dagger} \mathbf{A}^{-1}}{\mathbf{q}_{2}^{\dagger} \mathbf{A}^{-1} \tilde{\mathbf{p}}_{1}}+\left(\zeta_{2}-\zeta_{1}\right) \frac{\tilde{\mathbf{p}}_{1} \tilde{\mathbf{q}}_{2}^{\dagger} \mathbf{A}^{-2}}{\tilde{\mathbf{q}}_{2}^{\dagger} \mathbf{A}^{-2} \tilde{\mathbf{p}}_{1}}\right) \mathbf{A} . \\
& \mathbf{G}_{2}=\mathbf{A}\left(\left(z_{2}-z_{1}\right) \frac{\mathbf{p}_{1} \mathbf{q}_{2}^{\dagger}}{\mathbf{q}_{2}^{\dagger} \mathbf{p}_{1}}+\left(z_{1}-\zeta_{2}\right) \frac{\mathbf{A}^{-1} \tilde{\mathbf{p}}_{1} \mathbf{q}_{2}^{\dagger}}{\mathbf{q}_{2}^{\dagger} \mathbf{A}^{-1} \tilde{\mathbf{p}}_{1}}\right), \\
& \tilde{\mathbf{G}}_{2}=\mathbf{A}\left(\left(z_{2}-\zeta_{1}\right) \frac{\mathbf{A}^{-1} \mathbf{p}_{1} \tilde{\mathbf{q}}_{2}^{\dagger}}{\tilde{\mathbf{q}}_{2}^{\dagger} \mathbf{A}^{-1} \mathbf{p}_{1}}+\left(\zeta_{1}-\zeta_{2}\right) \frac{\mathbf{A}^{-2} \tilde{\mathbf{p}}_{1} \tilde{\mathbf{q}}_{2}^{\dagger}}{\tilde{\mathbf{q}}_{2}^{\dagger} \mathbf{A}^{-2} \tilde{\mathbf{p}}_{1}}\right),
\end{aligned}
$$

Proof. The equations in part (i) are obtained by taking the residues of the equation $\mathbf{B}_{2}^{\mathbf{A}}(z) \mathbf{B}_{1}^{\mathbf{A}}(z)=$ $\widetilde{\mathbf{B}}_{1}^{\mathbf{A}}(z) \widetilde{\mathbf{B}}_{2}^{\mathbf{A}}(z)$ at the points $z_{i}$ and the residues of the inverse equation at the points $\zeta_{i}$ and then equating the normalized row and column vectors of the resulting rank-one matrices. Using Lemma 2.1 we then obtain the equations in part (ii).

\section{The Isospectral Case}

We are now in the position to describe the equations of motion for the isospectral dynamics.

Theorem 3.1. Let $\underset{\sim}{\mathbf{Q}}=\left(\sim_{\sim}^{\left(\mathbf{p}_{1}\right.},{\underset{\sim}{2}}_{2}^{\dagger}\right), \mathbf{Q}=\left(\mathbf{p}_{1}, \mathbf{q}_{2}^{\dagger}\right), \tilde{\mathbf{Q}}=\left(\tilde{\mathbf{p}}_{1}, \tilde{\mathbf{q}}_{2}^{\dagger}\right)$, where each vector is considered modulo re-scaling,

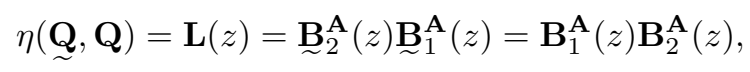

and

$$
\eta(\mathbf{Q}, \tilde{\mathbf{Q}})=\tilde{\mathbf{L}}(z)=\mathbf{B}_{2}^{\mathbf{A}}(z) \mathbf{B}_{1}^{\mathbf{A}}(z)=\tilde{\mathbf{B}}_{1}^{\mathbf{A}}(z) \tilde{\mathbf{B}}_{2}^{\mathbf{A}}(z) .
$$

Then

(i) The parameterization $\eta(\mathbf{Q}, \mathbf{Q})$ is given by

$$
\begin{aligned}
\eta(\underset{\sim}{\mathbf{Q}}, \mathbf{Q})=\mathbf{L}(z)= & \left(\mathbf{A}+\frac{1}{z-z_{1}}\left(\left(z_{1}-\zeta_{2}\right) \frac{\mathbf{p}_{1} \mathbf{q}_{2}^{\dagger}}{{\underset{\mathbf{q}}{2}}_{2}^{\dagger} \mathbf{A}^{-1} \mathbf{p}_{1}}+\left(\zeta_{2}-\zeta_{1}\right) \frac{\mathbf{p}_{1} \mathbf{q}_{2}^{\dagger} \mathbf{A}^{-1}}{\mathbf{q}_{2}^{\dagger} \mathbf{A}^{-2} \mathbf{p}_{1}}\right)\right) \times \\
& \left(\mathbf{A}+\frac{1}{z-z_{2}}\left(\left(z_{2}-\zeta_{1}\right) \frac{\stackrel{\mathbf{p}}{1}_{1} \mathbf{q}_{2}^{\dagger}}{\mathbf{q}_{2}^{\dagger} \mathbf{A}^{-1} \underline{\sim}_{1}}+\left(\zeta_{1}-\zeta_{2}\right) \frac{\mathbf{A}^{-1} \mathbf{p}_{1} \mathbf{q}_{2}^{\dagger}}{\mathbf{q}_{2}^{\dagger} \mathbf{A}^{-2} \mathbf{p}_{1}}\right)\right) .
\end{aligned}
$$

(ii) The equations of motion $(\mathbf{Q}, \tilde{\mathbf{Q}})=\Phi(\underset{\mathbf{Q}}{\mathbf{Q}})$ have the implicit form

$$
\begin{aligned}
& \left(\left(z_{2}-\zeta_{1}\right) \frac{\mathbf{A}^{-1} \underline{\sim}_{1}}{\mathbf{q}_{2}^{\dagger} \mathbf{A}^{-1} \underline{\sim}_{1}}+\left(\zeta_{1}-\zeta_{2}\right) \frac{\mathbf{A}^{-2} \mathbf{p}_{1}}{\mathbf{q}_{2}^{\dagger} \mathbf{A}^{-2} \mathbf{p}_{1}}\right)=\left(\left(z_{2}-z_{1}\right) \frac{\mathbf{p}_{1}}{\mathbf{q}_{2}^{\dagger} \mathbf{p}_{1}}+\left(z_{1}-\zeta_{2}\right) \frac{\mathbf{A}^{-1} \tilde{\mathbf{p}}_{1}}{\mathbf{q}_{2}^{\dagger} \mathbf{A}^{-1} \tilde{\mathbf{p}}_{1}}\right) \\
& \left(\left(z_{1}-\zeta_{2}\right) \frac{\mathfrak{q}_{2}^{\dagger} \mathbf{A}^{-1}}{{\underset{\mathbf{q}}{2}}_{2}^{\dagger} \mathbf{A}^{-1} \mathbf{p}_{1}}+\left(\zeta_{2}-\zeta_{1}\right) \frac{\mathbf{q}_{2}^{\dagger} \mathbf{A}^{-2}}{\mathbf{q}_{2}^{\dagger} \mathbf{A}^{-2} \mathbf{p}_{1}}\right)=\left(\left(z_{1}-z_{2}\right) \frac{\mathbf{q}_{2}^{\dagger}}{\mathbf{q}_{2}^{\dagger} \mathbf{p}_{1}}+\left(z_{2}-\zeta_{1}\right) \frac{\tilde{\mathbf{q}}_{2}^{\dagger} \mathbf{A}^{-1}}{\tilde{\mathbf{q}}_{2}^{\dagger} \mathbf{A}^{-1} \mathbf{p}_{1}}\right),
\end{aligned}
$$


and since we are only interested in the spaces spanned by $\tilde{\mathbf{p}}_{1}$ and $\tilde{\mathbf{q}}_{2}^{\dagger}$, we can take $\tilde{\mathbf{p}}_{1}$ and $\tilde{\mathbf{q}}_{2}^{\dagger}$ to be given by the explicit formulas

$$
\begin{aligned}
& \tilde{\mathbf{p}}_{1}=\mathbf{A}\left(\left(z_{1}-z_{2}\right) \frac{\mathbf{p}_{1}}{\mathbf{q}_{2}^{\dagger} \mathbf{p}_{1}}+\left(z_{2}-\zeta_{1}\right) \frac{\mathbf{A}^{-1} \underline{\mathbf{p}}_{1}}{\mathbf{q}_{2}^{\dagger} \mathbf{A}^{-1} \mathbf{p}_{1}}+\left(\zeta_{1}-\zeta_{2}\right) \frac{\mathbf{A}^{-2} \mathbf{p}_{1}}{\mathbf{q}_{2}^{\dagger} \mathbf{A}^{-2} \mathbf{p}_{1}}\right), \\
& \tilde{\mathbf{q}}_{2}^{\dagger}=\left(\left(z_{2}-z_{1}\right) \frac{\mathbf{q}_{2}^{\dagger}}{\mathbf{q}_{2}^{\dagger} \mathbf{p}_{1}}+\left(z_{1}-\zeta_{2}\right) \frac{\mathbf{q}_{2}^{\dagger} \mathbf{A}^{-1}}{{\underset{\mathbf{q}}{2}}_{2}^{\dagger} \mathbf{A}^{-1} \mathbf{p}_{1}}+\left(\zeta_{2}-\zeta_{1}\right) \frac{\mathbf{q}_{2}^{\dagger} \mathbf{A}^{-2}}{\mathbf{q}_{2}^{\dagger} \mathbf{A}^{-2} \mathbf{p}_{1}}\right) \mathbf{A} .
\end{aligned}
$$

(iii) Equations (3.1-3.2) are the discrete Euler-Lagrange equations (1.1) with the Lagrangian function

$$
\begin{aligned}
\mathcal{L}(\mathbf{X}, \mathbf{Y})= & \left(z_{2}-z_{1}\right) \log \left(\mathbf{x}_{2}^{\dagger} \mathbf{x}_{1}\right)+\left(z_{1}-\zeta_{2}\right) \log \left(\mathbf{x}_{2}^{\dagger} \mathbf{A}^{-1} \mathbf{y}_{1}\right)+ \\
& \left(\zeta_{2}-\zeta_{1}\right) \log \left(\mathbf{y}_{2}^{\dagger} \mathbf{A}^{-2} \mathbf{y}_{1}\right)+\left(\zeta_{1}-z_{2}\right) \log \left(\mathbf{y}_{2}^{\dagger} \mathbf{A}^{-1} \mathbf{x}_{1}\right),
\end{aligned}
$$

where $\mathbf{X}=\left(\mathbf{x}_{1}, \mathbf{x}_{2}^{\dagger}\right), \mathbf{Y}=\left(\mathbf{y}_{1}, \mathbf{y}_{2}^{\dagger}\right) \in \mathcal{Q}=\mathbb{P}^{r-1} \times\left(\mathbb{P}^{r-1}\right)^{\dagger}$. Every vector is an actual $r$-vector that we consider up to rescaling. This makes $\mathcal{L}$ defined up to an additive constant, but this does not affect the Euler-Lagrange equations.

Proof. Parts (i) follows immediately from equations (2.2-2.5) in Theorem 2.2. To establish parts (ii) and (iii),

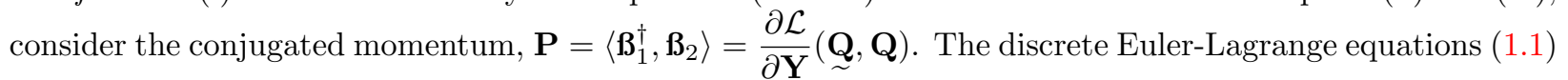
then split into two groups,

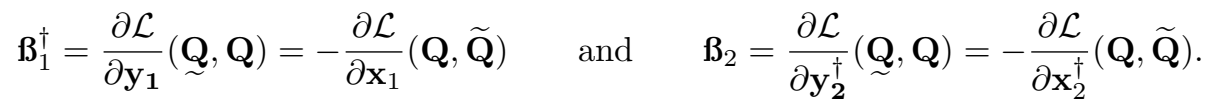

The first equation becomes

$$
\boldsymbol{\beta}_{1}^{\dagger}=\left(z_{1}-\zeta_{2}\right) \frac{\stackrel{\mathbf{q}}{2}_{2}^{\dagger} \mathbf{A}^{-1}}{{\underset{\mathbf{q}}{\dagger}}_{2}^{\dagger} \mathbf{A}^{-1} \mathbf{p}_{1}}+\left(\zeta_{2}-\zeta_{1}\right) \frac{\mathbf{q}_{2}^{\dagger} \mathbf{A}^{-2}}{\mathbf{q}_{2}^{\dagger} \mathbf{A}^{-2} \mathbf{p}_{1}}=\left(z_{1}-z_{2}\right) \frac{\mathbf{q}_{2}^{\dagger}}{\mathbf{q}_{2}^{\dagger} \mathbf{p}_{1}}+\left(z_{2}-\zeta_{1}\right) \frac{\tilde{\mathbf{q}}_{2}^{\dagger} \mathbf{A}^{-1}}{\tilde{\mathbf{q}}_{2}^{\dagger} \mathbf{A}^{-1} \mathbf{p}_{1}},
$$

which, on one hand, is equation (3.2), and on the other hand, is the equality of two different expression for $\mathbf{q}_{1}^{\dagger}$

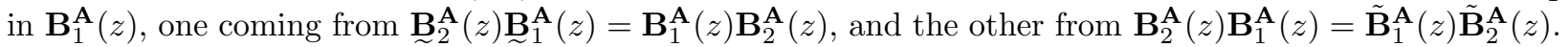
The other equation is similar, and that completes the proof.

\section{The Isomonodromic Case}

In this section we consider in detail an example of an elementary isomonodromy transformation defined in Section 3 of [Kri04]. We show that this transformation can be written in the Lagrangian form and then verify that, similarly to the polynomial case considered in [AB06], for rank $r=2$ matrices $\mathbf{L}(z)$ whose divisor has 2 simple zeroes and 2 simple poles, this transformation, when written in the spectral coordinates $p$ and $q$, reduces to the difference Painlevé equation dPV of the Sakai's hierarchy [Sak01]. Thus, we establish that $\mathrm{dPV}$ can be written in the Lagrangian form.

4.1. The spectral coordinates. Let $\mathbf{L}(z) \in \mathcal{M}_{r}^{\mathcal{D}}$, where $\mathcal{D}=\sum_{i}\left(z_{i}-\zeta_{i}\right)$, and all points are finite, distinct and do not differ by an integer. Since the isomonodromy equations (1.5) are invariant w.r.t. the conjugation action of the gauge group $\mathrm{GL}_{r}(\mathbb{C})$, we can use this action to diagonalize $\mathbf{L}_{0}, \mathbf{L}_{0}=\operatorname{diag}\left\{\rho_{1}, \ldots, \rho_{r}\right\}$, which reduces the gauge group to the subgroup $\mathrm{D}_{r} \subset \mathrm{GL}_{r}$ of the diagonal matrices. Next, consider some asymptotic properties of $\mathbf{L}(z)$. Namely, let us first introduce the matrix $\mathbf{L}_{\infty}:=-\operatorname{res}{ }_{\infty} \mathbf{L}(z) d z=\sum_{k} \mathbf{L}_{k}$ and put $k_{i}:=\frac{1}{\rho_{i}}\left(\mathbf{L}_{\infty}\right)_{i i}=\left(\mathbf{L}_{0}^{-1} \mathbf{L}_{\infty}\right)_{i i}$. Following [AB06], we define the type of $\mathbf{L}(z)$ as follows.

Definition 4.1. The type $\theta$ of the matrix $\mathbf{L}(z)$ is the following collection of parameters:

$$
\theta(\mathbf{L}(z))=\left\{z_{1}, \ldots, z_{n} ; \zeta_{1}, \ldots, \zeta_{n} ; \rho_{1}, \ldots, \rho_{r} ; k_{1}, \ldots, k_{r}\right\} .
$$

From the multiplicative representation (1.5) we see that these parameters are not independent, since

$$
k_{1}+\cdots+k_{r}=\operatorname{tr} \mathbf{L}_{0}^{-1} \mathbf{L}_{\infty}=\sum_{i=1}^{n} \operatorname{tr}\left(\mathbf{G}_{i} \mathbf{A}^{-1}\right)=\sum_{i=1}^{n}\left(z_{i}-\zeta_{i}\right) .
$$


For a general choice of parameters, this is the only relation. We denote by $\mathcal{M}_{r}^{\theta}$ the space of matrices of type $\theta$ and rank $r$. This space is clearly invariant under the conjugation by non-degenerate diagonal matrices. Factoring out this action we obtain the coarse moduli space $\widehat{\mathcal{M}}_{r}^{\theta}$.

It is worth mentioning that fixing the type $\theta$ of $\mathbf{L}(z)$ corresponds to considering a symplectic leaf of the canonical foliation of $\mathcal{M}_{r}^{\mathcal{D}}$ w.r.t. the universal algebro-geometric symplectic form $\omega$ of Krichever and Phong [KP97, KP98], see the survey [DKP02] for details.

Lemma 4.1. The dimension of the big cell of the moduli space $\widehat{\mathcal{M}}_{r}^{\theta}$ is

$$
\operatorname{dim} \mathcal{M}_{r}^{\theta}=2(n-1)(r-1) .
$$

Proof. Consider the multiplicative representation of $\mathbf{L}(z)$. Each elementary divisor is given by $2(r-1)$ parameters. Fixing the diagonal elements of $\mathbf{L}_{\infty}$ imposes $r-1$ (in view of (4.1)) additional conditions, and therefore

$$
\operatorname{dim} \mathcal{M}_{r}^{\theta}=n(2 r-2)-(r-1)=(2 n-1)(r-1) .
$$

Further action by the diagonal matrices reduces the dimension by $r-1$, and so

$$
\operatorname{dim} \widehat{\mathcal{M}}_{r}^{\theta}=2(n-1)(r-1) .
$$

Thus, when $n=r=2, \widehat{\mathcal{M}}_{r}^{\theta}$ is a complex surface. The spectral coordinate system $(q, p)$ on the space $\widehat{\mathcal{M}}_{2}^{\theta}$ is given by the zero $q$ of $\mathbf{L}(z)_{12}$ and the value of $\mathbf{L}(z)_{11}$ at $q$ normalized in the following way:

$$
p=\frac{q-z_{1}}{q-\zeta_{2}} \mathbf{L}(q)_{11}, \quad \text { where } \quad \mathbf{L}(q)_{12}=0 .
$$

The spectral coordinates $p$ and $q$ are essentially the same as in [AB06]. From the integrable systems point of view these coordinates are a particular case of the Darboux coordinates of the universal algebrogeometric symplectic form $\omega$. For a particular case of the hyperelliptic KdV curves these coordinates were first considered by Novikov and Veselov [VN82], the general case was recently established by Krichever in [Kri00a, Kri00b].

Note that since

$$
\operatorname{det} \mathbf{L}(z)=\operatorname{det} \mathbf{L}_{0} \frac{\left(z-\zeta_{1}\right)\left(z-\zeta_{2}\right)}{\left(z-z_{1}\right)\left(z-z_{2}\right)}, \quad \mathbf{L}(q)=\left[\begin{array}{cc}
\frac{p\left(q-\zeta_{2}\right)}{\left(q-z_{1}\right)} & 0 \\
* & \frac{\rho_{1} \rho_{2}\left(q-\zeta_{1}\right)}{p\left(q-z_{2}\right)}
\end{array}\right]
$$

Next, we need to obtain the explicit formulas for the additive and the multiplicative representations of $\mathbf{L}(z)$ in the spectral coordinates.

\subsection{The Additive and the Multiplicative Representations of $\mathbf{L}(z)$.}

Lemma 4.2. The additive representation (1.4) of $\mathbf{L}(z)$ in the pq-coordinates is given by

$$
\begin{aligned}
& \mathbf{L}_{1}=\frac{q-z_{1}}{z_{2}-z_{1}}\left[\begin{array}{c}
1 \\
\rho_{2}\left(q-z_{2}+k_{2}\right)-\frac{\rho_{1} \rho_{2}}{p}\left(q-\zeta_{1}\right)
\end{array}\right]\left[\rho_{1}\left(q-z_{2}+k_{1}\right)-\frac{p\left(q-z_{2}\right)\left(q-\zeta_{2}\right)}{q-z_{1}} 1\right],
\end{aligned}
$$

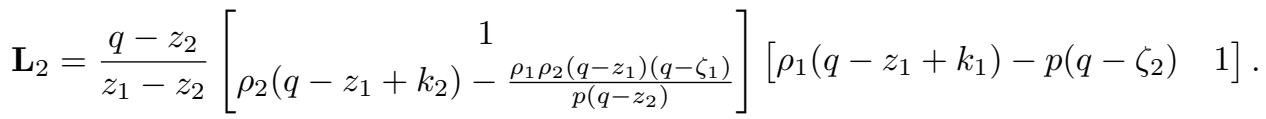

Proof. By choosing a linear normalization in which the first components of the column vectors and the second components of the row vectors are equal to 1 , we get

$$
\mathbf{L}(z)=\left[\begin{array}{cc}
\rho_{1} & 0 \\
0 & \rho_{2}
\end{array}\right]+\alpha_{1} \frac{\left[\begin{array}{c}
1 \\
a^{1}
\end{array}\right]\left[\begin{array}{ll}
b_{1} & 1
\end{array}\right]}{z-z_{1}}+\alpha_{2} \frac{\left[\begin{array}{c}
1 \\
a^{2}
\end{array}\right]\left[\begin{array}{ll}
b_{2} & 1
\end{array}\right]}{z-z_{2}} .
$$


We then obtain the following equations for $\alpha_{1}, \alpha_{2}, b_{1}, b_{2}$ :

$$
\begin{aligned}
\mathbf{L}(q)_{12} & =\frac{\alpha_{1}}{q-z_{1}}+\frac{\alpha_{2}}{q-z_{2}}=0 \\
p & =\frac{q-z_{1}}{q-\zeta_{2}} \mathbf{L}(q)_{11}=\frac{q-z_{1}}{q-\zeta_{2}}\left(\rho_{1}+\frac{\alpha_{1}}{q-z_{1}} b_{1}+\frac{\alpha_{2}}{q-z_{2}} b_{2}\right) \\
& =\frac{q-z_{1}}{q-\zeta_{2}}\left(\rho_{1}+\frac{\alpha_{1}}{q-z_{1}}\left(b_{1}-b_{2}\right)\right), \\
\left(\mathbf{L}_{\infty}\right)_{11} & =\alpha_{1} b_{1}+\alpha_{2} b_{2}=\rho_{1} k_{1} .
\end{aligned}
$$

Note that we have only three equation for the four unknowns. This is due to the conjugation action by the constant diagonal matrices $\mathbf{D}=\operatorname{diag}\left\{\gamma_{1}, \gamma_{2}\right\}$. Taking this action into account,

$$
\mathbf{D}\left(\alpha_{i} \mathbf{a}_{i} \mathbf{b}_{i}^{\dagger}\right) \mathbf{D}^{-1}=\left(\frac{\gamma_{1}}{\gamma_{2}} \alpha_{i}\right)\left[\begin{array}{c}
1 \\
\left(\frac{\gamma_{2}}{\gamma_{1}} a^{i}\right)
\end{array}\right]\left[\left(\frac{\gamma_{2}}{\gamma_{1}} b_{i}\right) \quad 1\right]
$$

we see that we can provisionally put $\alpha_{1}=1$. Then

$$
\begin{aligned}
\alpha_{2} & =-\frac{q-z_{2}}{q-z_{1}} \\
b_{1} & =\rho_{1} k_{1}-\alpha_{2} b_{2}=\rho_{1} k_{1}+\frac{q-z_{2}}{q-z_{1}} b_{2}=b_{2}+\left(q-\zeta_{2}\right) p-\left(q-z_{1}\right) \rho_{1},
\end{aligned}
$$

and so

$$
\begin{aligned}
& b_{2}=\frac{q-z_{1}}{z_{2}-z_{1}}\left(\rho_{1}\left(q-z_{1}+k_{1}\right)-p\left(q-\zeta_{2}\right)\right) \\
& b_{1}=\frac{q-z_{1}}{z_{2}-z_{1}}\left(\rho_{1}\left(q-z_{2}+k_{1}\right)-p \frac{\left(q-\zeta_{2}\right)\left(q-z_{2}\right)}{q-z_{1}}\right) .
\end{aligned}
$$

To find $a^{1}$ and $a^{2}$ we use the definition of $k_{2}$ and $\operatorname{det} \mathbf{L}(q)$ :

$$
\begin{aligned}
\left(\mathbf{L}_{\infty}\right)_{22} & =\alpha_{1} a^{1}+\alpha_{2} a^{2}=\rho_{2} k_{2} \\
\operatorname{det} \mathbf{L}(q) & =\operatorname{det}\left[\begin{array}{cc}
\frac{q-\zeta_{2}}{q-z_{1}} p & 0 \\
* & \rho_{2}+\frac{\alpha_{1}}{q-z_{1}} a^{1}+\frac{\alpha_{2}}{q-z_{2}} a^{2}
\end{array}\right]=p \frac{q-\zeta_{2}}{q-z_{1}}\left(\rho_{2}+\frac{a^{1}-a^{2}}{q-z_{1}}\right) \\
& =\rho_{1} \rho_{2} \frac{\left(q-\zeta_{1}\right)\left(q-\zeta_{2}\right)}{\left(q-z_{1}\right)\left(q-z_{2}\right)}
\end{aligned}
$$

and so

$$
a^{1}-a^{2}=\frac{\rho_{1} \rho_{2}}{p} \cdot \frac{\left(q-\zeta_{1}\right)\left(q-z_{1}\right)}{q-z_{2}}-\rho_{2}\left(q-z_{1}\right)
$$

Thus,

$$
a^{1}=\rho_{2} k_{2}-\alpha_{2} a^{2}=\rho_{2} k_{2}+\frac{q-z_{2}}{q-z_{1}} a^{2}=a^{2}+\frac{\rho_{1} \rho_{2}}{p} \cdot \frac{\left(q-\zeta_{1}\right)\left(q-z_{1}\right)}{q-z_{2}}-\rho_{2}\left(q-z_{1}\right),
$$

which results in

$$
\begin{aligned}
& a^{1}=\frac{q-z_{1}}{z_{2}-z_{1}}\left(\rho_{2}\left(q-z_{2}+k_{2}\right)-\frac{\rho_{1} \rho_{2}\left(q-\zeta_{1}\right)}{p}\right) \\
& a^{2}=\frac{q-z_{1}}{z_{2}-z_{1}}\left(\rho_{2}\left(q-z_{1}+k_{2}\right)-\frac{\rho_{1} \rho_{2}}{p} \cdot \frac{\left(q-\zeta_{1}\right)\left(q-z_{1}\right)}{q-z_{2}}\right) .
\end{aligned}
$$

Conjugating by the diagonal matrix $\mathbf{D}=\operatorname{diag}\left\{q-z_{1}, z_{2}-z_{1}\right\}$ finishes the proof.

To obtain the multiplicative description of $\mathbf{L}(z)$ in the spectral coordinates, we need the following Lemma. 
Lemma 4.3. Let

$$
\mathbf{L}(z)=\mathbf{L}_{0}+\frac{\mathbf{L}_{1}}{z-z_{1}}+\frac{\mathbf{L}_{2}}{z-z_{2}}=\mathbf{B}_{1}^{\mathbf{A}}(z) \mathbf{B}_{2}^{\mathbf{A}}(z),
$$

where $\mathbf{L}_{0}=\mathbf{A}^{2}$. Then

(i) the additive representation is given in terms of the multiplicative representation by

$$
\mathbf{L}_{1}=\mathbf{G}_{1} \mathbf{B}_{2}^{\mathbf{A}}\left(z_{1}\right), \quad \mathbf{L}_{2}=\mathbf{B}_{1}^{\mathbf{A}}\left(z_{2}\right) \mathbf{G}_{2}
$$

(ii) the multiplicative representation is given in terms of the additive representation by

$$
\mathbf{G}_{1} \mathbf{A}=\mathbf{L}_{1}+\frac{\left(z_{1}-\zeta_{1}\right)-\operatorname{tr}\left(\mathbf{L}_{0}^{-1} \mathbf{L}_{1}\right)}{\operatorname{tr}\left(\mathbf{L}_{0}^{-1} \mathbf{L}_{1} \mathbf{L}_{2}\right)} \mathbf{L}_{1} \mathbf{L}_{2}, \quad \mathbf{A G}_{2}=\mathbf{L}_{2}+\frac{\left(z_{2}-\zeta_{2}\right)-\operatorname{tr}\left(\mathbf{L}_{0}^{-1} \mathbf{L}_{2}\right)}{\operatorname{tr}\left(\mathbf{L}_{0}^{-1} \mathbf{L}_{1} \mathbf{L}_{2}\right)} \mathbf{L}_{1} \mathbf{L}_{2} .
$$

Proof. Part (i) follows immediately from taking the residues at $z_{i}$. To establish part (ii), note that from part (i) it follows that

$$
\mathbf{G}_{1} \mathbf{A}=\mathbf{L}_{1}+\frac{\mathbf{G}_{1} \mathbf{G}_{2}}{z_{2}-z_{1}}, \quad \mathbf{A G}_{2}=\mathbf{L}_{2}+\frac{\mathbf{G}_{1} \mathbf{G}_{2}}{z_{1}-z_{2}}
$$

and so what we really need is to establish the identity

$$
\mathbf{G}_{1} \mathbf{G}_{2}=\left(z_{2}-z_{1}\right) \frac{\left(z_{1}-\zeta_{1}\right)-\operatorname{tr}\left(\mathbf{L}_{0}^{-1} \mathbf{L}_{1}\right)}{\operatorname{tr}\left(\mathbf{L}_{0}^{-1} \mathbf{L}_{1} \mathbf{L}_{2}\right)} \mathbf{L}_{1} \mathbf{L}_{2}=\left(z_{1}-z_{2}\right) \frac{\left(z_{2}-\zeta_{2}\right)-\operatorname{tr}\left(\mathbf{L}_{0}^{-1} \mathbf{L}_{2}\right)}{\operatorname{tr}\left(\mathbf{L}_{0}^{-1} \mathbf{L}_{1} \mathbf{L}_{2}\right)} \mathbf{L}_{1} \mathbf{L}_{2} .
$$

Since $\mathbf{L}_{i}$ and $\mathbf{G}_{i}$ are of rank one, from (i) we see that $\mathbf{G}_{1} \mathbf{G}_{2}=\gamma \mathbf{L}_{1} \mathbf{L}_{2}$. The proportionality constant $\gamma$ can be found using either of the equations (4.2). For example, using the first equation, we see that

$$
\gamma=\frac{\operatorname{tr}\left(\mathbf{G}_{1} \mathbf{G}_{2} \mathbf{A}^{-2}\right)}{\operatorname{tr}\left(\mathbf{L}_{0}^{-1} \mathbf{L}_{1} \mathbf{L}_{2}\right)}=\frac{\left(z_{2}-z_{1}\right) \operatorname{tr}\left(\mathbf{G}_{1} \mathbf{A}^{-1}-\mathbf{L}_{1} \mathbf{A}^{-2}\right)}{\operatorname{tr}\left(\mathbf{L}_{0}^{-1} \mathbf{L}_{1} \mathbf{L}_{2}\right)}=\left(z_{2}-z_{1}\right) \frac{\left(z_{1}-\zeta_{1}\right)-\operatorname{tr}\left(\mathbf{L}_{0}^{-1} \mathbf{L}_{1}\right)}{\operatorname{tr}\left(\mathbf{L}_{0}^{-1} \mathbf{L}_{1} \mathbf{L}_{2}\right)} .
$$

Corollary 4.4. The multiplicative representation of $\mathbf{L}(z)$ in the spectral coordinates is given by

$$
\mathbf{L}(z)=\left(\mathbf{L}_{0}+\frac{\mathbf{G}_{1} \mathbf{A}}{z-z_{1}}\right) \mathbf{L}_{0}^{-1}\left(\mathbf{L}_{0}+\frac{\mathbf{A G}_{2}}{z-z_{2}}\right)
$$

where

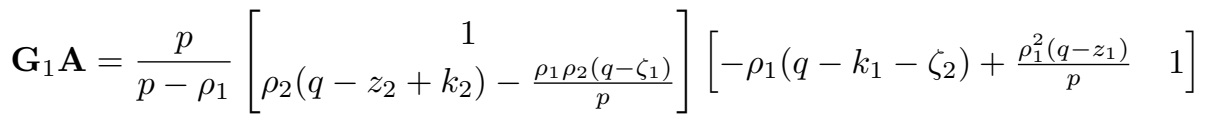

$$
\begin{aligned}
& \mathbf{A G}_{2}=\frac{\rho_{1}}{\rho_{1}-p}\left[\begin{array}{c}
1 \\
-\rho_{2}\left(q-k_{2}-\zeta_{1}\right)+\frac{\rho_{2} p}{\rho_{1}}\left(q-z_{2}\right)
\end{array}\right]\left[\rho_{1}\left(q+k_{1}-z_{1}\right)-p\left(q-\zeta_{2}\right) \quad 1\right] .
\end{aligned}
$$

Proof. Proof is a direct calculation.

4.3. The isomonodromic Lagrangian and $\mathbf{d P V}$. We are now ready to prove the main Theorem of this section.

Theorem 4.5. Consider the special isomonodromic transformation of the form

$$
\mathbf{L}(z)=\mathbf{B}_{1}^{\mathbf{A}}(z) \mathbf{B}_{2}^{\mathbf{A}}(z) \mapsto \tilde{\mathbf{L}}(z)=\tilde{\mathbf{B}}_{1}^{\mathbf{A}}(z) \tilde{\mathbf{B}}_{2}^{\mathbf{A}}(z)=\mathbf{B}_{2}^{\mathbf{A}}(z+1) \mathbf{B}_{1}^{\mathbf{A}}(z)=\mathbf{B}_{2}^{\mathbf{A}}(z+1) \mathbf{L}(z)\left(\mathbf{B}_{2}^{\mathbf{A}}(z)\right)^{-1} .
$$

(i) This transformation satisfies the time-dependent Euler-Lagrange equations

$$
\frac{\partial \mathcal{L}}{\partial \mathbf{Y}}\left(\mathbf{Q}_{k-1}, \mathbf{Q}_{k}, k-1\right)+\frac{\partial \mathcal{L}}{\partial \mathbf{X}}\left(\mathbf{Q}_{k}, \mathbf{Q}_{k+1}, k\right)=0
$$

with the Lagrangian function given by

$$
\begin{aligned}
\mathcal{L}(\mathbf{X}, \mathbf{Y}, t)= & \left(z_{2}(t)-z_{1}\right) \log \left(\mathbf{x}_{2}^{\dagger} \mathbf{x}_{1}\right)+\left(z_{1}-\zeta_{2}(t)\right) \log \left(\mathbf{x}_{2}^{\dagger} \mathbf{A}^{-1} \mathbf{y}_{1}\right)+ \\
& \left(\zeta_{2}(t)-\zeta_{1}\right) \log \left(\mathbf{y}_{2}^{\dagger} \mathbf{A}^{-2} \mathbf{y}_{1}\right)+\left(\zeta_{1}-z_{2}(t)\right) \log \left(\mathbf{y}_{2}^{\dagger} \mathbf{A}^{-1} \mathbf{x}_{1}\right),
\end{aligned}
$$

where $z_{2}(t)=z_{2}-t$ and $\zeta_{2}(t)=\zeta_{2}-t$. 
(ii) This transformation defines a birational map $\psi: \widehat{\mathcal{M}}_{2}^{\theta} \rightarrow \widehat{\mathcal{M}}_{2}^{\tilde{\theta}}$, where $\tilde{z}_{2}=z_{2}-1, \tilde{\zeta}_{2}=\zeta_{2}-1$ and all other parameters are unchanged. In the spectral coordinates $p, q$ on $\widehat{\mathcal{M}}_{2}^{\theta}$ and $\tilde{p}$, $\tilde{q}$ on $\widehat{\mathcal{M}}_{2}^{\tilde{\theta}}$ this transformation is given by the difference Painleve equation $d P V$ of the Sakai's hierarchy,

$$
\left\{\begin{array}{c}
q+\tilde{q}=z_{2}+\tilde{\zeta_{2}}-\frac{\rho_{1}\left(k_{2}+\zeta_{1}-z_{2}\right)}{p-\rho_{1}}+\frac{\rho_{2}\left(k_{2}+\tilde{\zeta}_{2}-z_{1}\right)}{p-\rho_{2}} \\
p \tilde{p}=\rho_{1} \rho_{2} \frac{\left(\tilde{q}-\zeta_{1}\right)\left(\tilde{q}-z_{1}\right)}{\left(\tilde{q}-\tilde{\zeta}_{2}\right)\left(\tilde{q}-\tilde{z}_{2}\right)}
\end{array} .\right.
$$

Proof. Part (i) is proved in exactly the same way as Theorem 3.1 part (iii). To establish part (ii), it suffices to rewrite equation (4.3) in the form $\left(\mathbf{B}_{2}^{\mathbf{A}}(z+1)\right)^{-1} \tilde{\mathbf{L}}(z)=\mathbf{B}_{1}^{\mathbf{A}}(z)$ and then evaluate it at $\tilde{q}$. From the definition of $\tilde{q}$ and $\tilde{p}$, we get

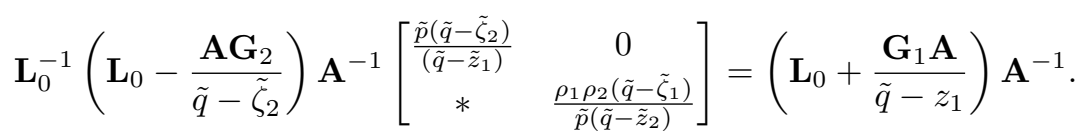

Thus, second columns of the matrices $\mathbf{L}_{0}^{-1}\left(\mathbf{L}_{0}-\frac{\mathbf{A G}_{2}}{\tilde{q}-\tilde{\zeta}_{2}}\right)$ and $\left(\mathbf{L}_{0}+\frac{\mathbf{G}_{1} \mathbf{A}}{\tilde{q}-z_{1}}\right)$ are proportional with the proportionality coefficient $\frac{\rho_{1} \rho_{2}\left(\tilde{q}-\tilde{\zeta}_{1}\right)}{\tilde{p}\left(\tilde{q}-\tilde{z}_{2}\right)}$. Direct calculation then completes the proof.

I am very grateful to I. Krichever for many helpful and stimulating discussions. This research was supported in part by the University of Northern Colorado Summer 2006 SPARC Small Grant Assistance Program.

\section{REFERENCES}

[AB06] D. Arinkin and A. Borodin, Moduli spaces of d-connections and difference Painlevé equations, Duke Math. J. 134 (2006), no. 3, 515-556. MR MR2254625 (2007h:14047)

[AB07] D Arinkin and A. Borodin, Tau-function of discrete isomonodromy transformations and probability, 2007.

[BD02] Alexei Borodin and Percy Deift, Fredholm determinants, Jimbo-Miwa-Ueno $\tau$-functions, and representation theory, Comm. Pure Appl. Math. 55 (2002), no. 9, 1160-1230. MR 1908746

[Bir11] George D. Birkhoff, General theory of linear difference equations, Trans. Amer. Math. Soc. 12 (1911), no. 2, $243-284$. MR 1500888

[Bor03] Alexei Borodin, Discrete gap probabilities and discrete Painlevé equations, Duke Math. J. 117 (2003), no. 3, 489-542. MR MR1979052 (2004g:39030)

[Bor04] _ Isomonodromy transformations of linear systems of difference equations, Ann. of Math. (2) 160 (2004), no. 3 , 1141-1182. MR MR2144976

[DKP02] Eric D'Hoker, I. M. Krichever, and D. H. Phong, Seiberg-Witten theory, symplectic forms, and hamiltonian theory of solitons, 122002.

[DLT89] P. Deift, L. C. Li, and C. Tomei, Matrix factorizations and integrable systems, Comm. Pure Appl. Math. 42 (1989), no. 4, 443-521. MR MR990138 (92a:58062)

[GR04] B. Grammaticos and A. Ramani, Discrete Painlevé equations: a review, Discrete integrable systems, Lecture Notes in Phys., vol. 644, Springer, Berlin, 2004, pp. 245-321. MR MR2087743 (2005g:39032)

[KP97] I. M. Krichever and D. H. Phong, On the integrable geometry of soliton equations and $N=2$ supersymmetric gauge theories, J. Differential Geom. 45 (1997), no. 2, 349-389. MR MR1449977 (98b:58078)

[KP98] - Symplectic forms in the theory of solitons, Surveys in differential geometry: integral systems [integrable systems], Surv. Differ. Geom., IV, Int. Press, Boston, MA, 1998, pp. 239-313. MR MR1726930 (2001k:37114)

[Kri] I. Krichever, Private communications.

[Kri00a] _ Elliptic analog of the Toda lattice, Internat. Math. Res. Notices (2000), no. 8, 383-412. MR MR1753690 (2001e:14033)

[Kri00b] _ Elliptic solutions to difference nonlinear equations and nested Bethe ansatz equations, Calogero-MoserSutherland models (Montréal, QC, 1997), CRM Ser. Math. Phys., Springer, New York, 2000, pp. 249-271. MR MR1843575 (2002h:37146)

[Kri04] I. M. Krichever, Analytic theory of difference equations with rational and elliptic coefficients and the Riemann-Hilbert problem, Uspekhi Mat. Nauk 59 (2004), no. 6, 117-1154.

[MV91] Jürgen Moser and Alexander P. Veselov, Discrete versions of some classical integrable systems and factorization of matrix polynomials, Comm. Math. Phys. 139 (1991), no. 2, 217-243. MR MR1120138 (92g:58054)

[MW01] J. E. Marsden and M. West, Discrete mechanics and variational integrators, Acta Numer. 10 (2001), $357-514$. MR MR2009697 (2004h:37130) 
[Sak01] Hidetaka Sakai, Rational surfaces associated with affine root systems and geometry of the Painlevé equations, Comm. Math. Phys. 220 (2001), no. 1, 165-229. MR MR1882403 (2003c:14030)

[Sur03] Yuri B. Suris, The problem of integrable discretization: Hamiltonian approach, Progress in Mathematics, vol. 219, Birkhäuser Verlag, Basel, 2003. MR MR1993935 (2004h:37086)

[Sym82] W. W. Symes, The $Q R$ algorithm and scattering for the finite nonperiodic Toda lattice, Phys. D 4 (1981/82), no. 2, 275-280. MR MR653781 (83h:58053)

[Ves88] A. P. Veselov, Integrable systems with discrete time, and difference operators, Funktsional. Anal. i Prilozhen. 22 (1988), no. 2, 1-13, 96. MR MR947601 (90a:58081)

[Ves91] , Integrable Lagrangian correspondences and factorization of matrix polynomials, Funktsional. Anal. i Prilozhen. 25 (1991), no. 2, 38-49, 96. MR MR1142207 (93b:58081)

[VN82] A. P. Veselov and S. P. Novikov, Poisson brackets that are compatible with the algebraic geometry and the dynamics of the Korteweg-de Vries equation on the set of finite-gap potentials, Dokl. Akad. Nauk SSSR 266 (1982), no. 3, 533-537. MR MR672377 (84j:58065)

School of Mathematical Sciences, University of Northern Colorado, Greeley, CO 80639

E-mail address: adzham@unco.edu

$U R L$ : www. unco.edu/adzham 